\title{
Does manufacturers' size affect the prevalence of mycobiota and occurrence of mycotoxins in spices and spice-based products?
}

\begin{abstract}
The present work aimed to establish the prevalence of mycobiota and occurrence of mycotoxins (aflatoxins and ochratoxin A) in spices and spice-based products, and correlate these to their manufacturers' sizes. A total of 90 spice, sauce and paste samples were purchased; 3 manufacturer sizes (small, medium, large) $\times 3$ types of samples (spices, sauces, pastes) $\times 5$ brands $\times 2$ replicates. The prevalence of mycobiota was assessed with dichloran rose bengal chloramphenicol (DRBC) and Aspergillus flavus and Aspergillus parasiticus (AFPA) medium, while the occurrence of mycotoxins was quantified with HPLC-FLD. Large-scale manufacturers were found to adopt a greater number of safety and quality certifications. Small-scale manufacturers significantly yielded the highest total fungal loads on DRBC $(\log 5.084 \pm 0.417$ $\mathrm{cfu} / \mathrm{g}$ paste, $\log 6.253 \pm 0.407 \mathrm{cfu} / \mathrm{g}$ sauce, $\log 6.662 \pm 0.222 \mathrm{cfu} / \mathrm{g}$ spice) and AFPA (log $4.461 \pm 0.451 \mathrm{cfu} / \mathrm{g}$ paste, $\log 5.661 \pm 0.395 \mathrm{cfu} / \mathrm{g}$ sauce, and $\log 6.269 \pm 0.432 \mathrm{cfu} / \mathrm{g}$ spice). Correlation analysis (Pearson's r) revealed that manufacturers' sizes positively influenced (DRBC r=0.781; AFPA r=0.702) the prevalence of mycobiota. Aflatoxin B1 was present in 6/30 (20\%) paste samples, $1 / 30$ sauce samples $(3.33 \%)$ and $12 / 30$ spice samples (40\%). Aflatoxin B2 was only present in $2 / 30$ sauce samples $(6.67 \%)$. Aflatoxin G1 and G2 were absent from all samples. Ochratoxin A was present in 11/30 (36.67\%) paste samples, 5/30 sauce samples $(16.67 \%)$ and $21 / 30$ spice samples $(70 \%)$. It was found that, to a certain extent, the size of and certification adopted by manufacturers affected the prevalence of mycobiota and the occurrence of mycotoxins in spices and spice-based products analysed in the present work. Nevertheless, it is henceforth recommended that a surveillance study of this nature be extended and widened in terms of number of samples as well as type of spices, sauces and pastes to obtain a more thorough and significant profile of the products' food safety and quality level.
\end{abstract}

Keyword: Aflatoxins; Ochratoxin A; Chilli; Pepper; Pastes 